\title{
AN ELASTIC MODEL FOR LAGRANGIAN TURBULENCE AND DIFFUSION
}

\author{
M.S. El NASChie \\ Cornell University, Sibley School of Mechanical and Aerospace Engineering, \\ Ithaca N.Y. 14853, USA \\ (Received Juni 25, 1990)
}

\begin{abstract}
Using a special analogy to a parametrically forced mathematical pendulum with energy flux, it is attempted here to model the vortex wake in a fluid due to the movement of a cylindrical solid body. We also show that the path taken by certain fluid particles represents a homoclinic soliton and corresponds to a spacial separatrix. This in turn may lead through deterministic fluctuation to diffusion-like chaotic motion.
\end{abstract}

PACS numbers: $03.40 . \mathrm{Gc}$

\section{Introduction}

In contrast to the evolution of the velocity field of a fluid which is governed by Navier-Stokes partial differential equation, the trajectory of a fluid particle is governed by an ordinary differential equation. Consequently in a Lagrangian description of a fluid, the results of dynamical system theory of autonomous O.D.E. may be used directly. This way the observed randomness of a Lagrangian turbulence might be interpreted as deterministic chaos [1]. In the present work we give simple arguments based upon a classical elastic model to confirm the existence of chaotic diffusion-like particle paths in the presence of deterministic wave-like fluctuation. There are numerous analogies between elastomechanical and hydrodynamical problems such as that holding between the shape of a free fluid surface. under surface tension and the bending of an elastic wire. In what follows, we use another analogy relating to the Euler elastica [2-6] and the lateral displacement of a fluid particle due to the motion of a circular cylindrical solid body in a two-dimensional flow $[7,8]$.

Subsequently we modify our elastica model in such a way that allows us to mimic to some reasonable extent the well-known Kàrmàn vortices street created 
in the fluid by movement of the cylinder at certain Reynold numbers [8] (Fig. 4, $6)$. Finally the similarity of these vortices to travelling loop solitons $[9,10]$ and the cornu-like spiral chaos which arises in Riemann sums approximating oscillatory integrals [11] is pointed out (Fig. 5).

\section{Pseudo-random walk of a fluid particle}

Consider a circular cylinder of radius a moving in a liquid when its centre is at the origin of a fixed Cartesian system $(x, y)$. It is easily shown that the curvature of the path of a fluid particle which is displaced laterly by the cylinder is given by (see Appendix 1):

$$
\kappa=\frac{2}{a^{2}}(2 y-\eta)
$$

Noting that $\kappa=\Phi^{\prime}=\mathrm{d} \Phi / \mathrm{d} s$, where $s$ is the arch length of the path and $\Phi$ is the slope of the path, one could differentiate the previous equation once and find that

$$
\Phi^{\prime \prime}=\left(\frac{4}{a^{2}}\right)\left(\frac{\mathrm{d} y}{\mathrm{~d} s}\right),
$$

Since $\mathrm{d} y / \mathrm{d} s=\sin \Phi$ the previous equation is obviously identical to the classical elastica equation of Euler (see Appendix 2). Consequently we may state that these looped stereophoid-like paths which were drawn long ago by J. Maxwell and G. Taylor $[12,13]$ are actually spacial homoclinic orbits. In other words they are spacial separatrix corresponding in a dynamical analogy to a homoclinic orbit in phase space $[4,6,14]$. It follows then that the entire chain of reasoning used previously in establishing the possibility for statical loop soliton chaos in the Eulcr elastica may now be carried over to show that deterministic wave-like fluctuation could lead in the appropriate region of parametric and initial values to a completely chaotic particle path. The loops themselves may still persist but their spacial distribution will be erratic and will differ essentially from the classical picture given in text books. Now, due to the intimate relationship between the stability of orbits with errational wending numbers, damping and random walker, we feel that the preceding discussion might be relevant for a new interpretation of diffusion-like process [15].

It might even be that the analogy with the elastica can be taken one step further using the analogy between the elastica and the elastic circular ring under external pressure $[14,16,17]$. That way we may expect that circular motion of a circular cylinder in a fluid will produce similar diffusion-like behaviour of the fluid particles. However, what may be even more interesting is what could happen if we have vortices in the fluid. This we discuss next.

\section{Chaotic vortices, Cornu spirals and the fluttering elastica}

In the previous Section we considered the effect of a cylinder moving in a fluid. Here we consider two circular cylinders streamed by a fluid [8]. In this case, 


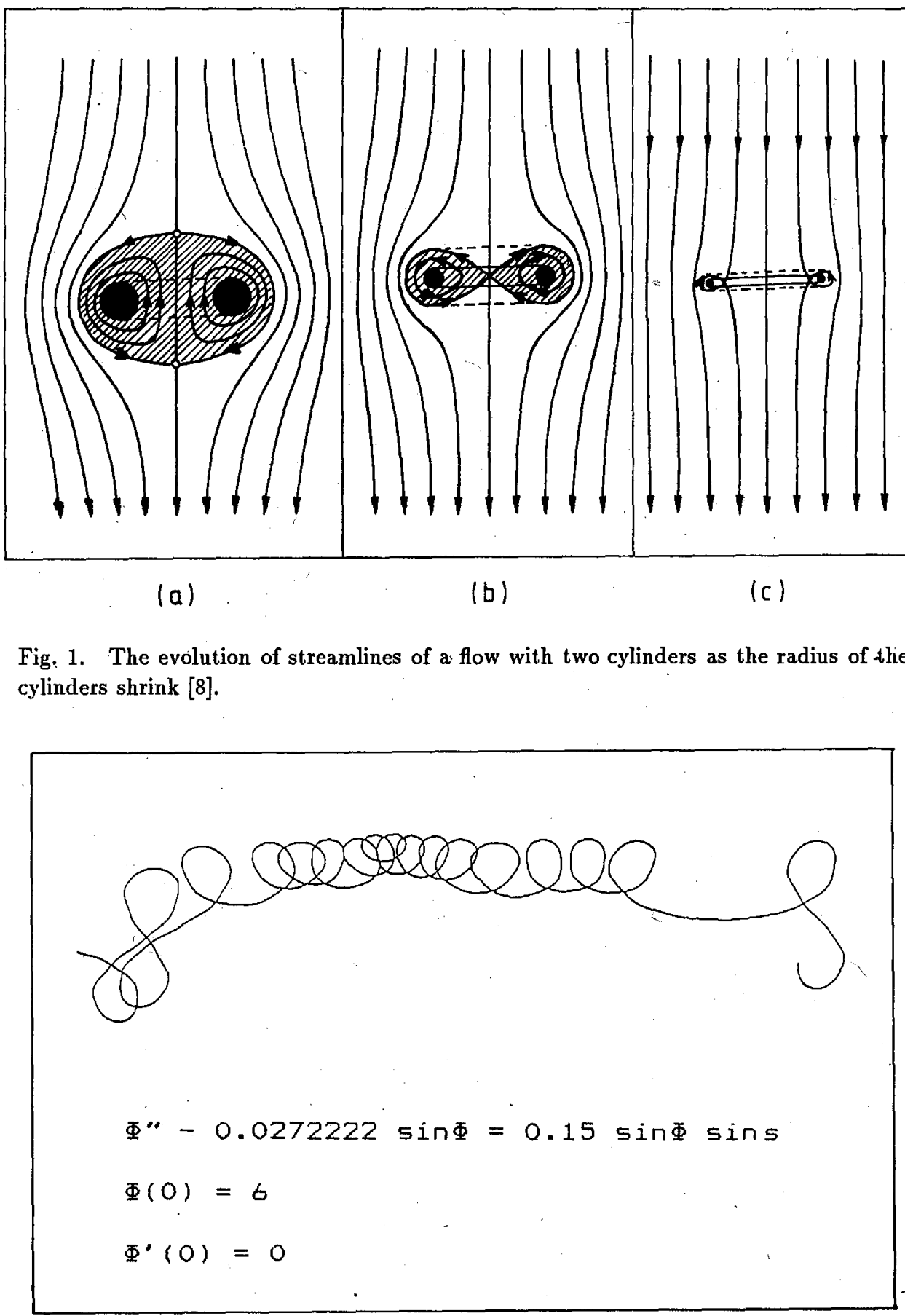

Fig. 2. Spacial soliton loop chaos of the imperfect Hamiltonian elastica. 
as is well known, two kinds of streamlines form as shown in Fig. 1(a). Now as we let the radius of the cylinder shrink we obtain the sequence shown in Fig. 1(b,c). Anyone who has observed the motion of a travelling loop soliton in a long flexible wire $[6,9]$ will notice the similarity between them and the vortices shown in Fig. 1(c) which can easily be made visible in an actual experiment. It is this similarity

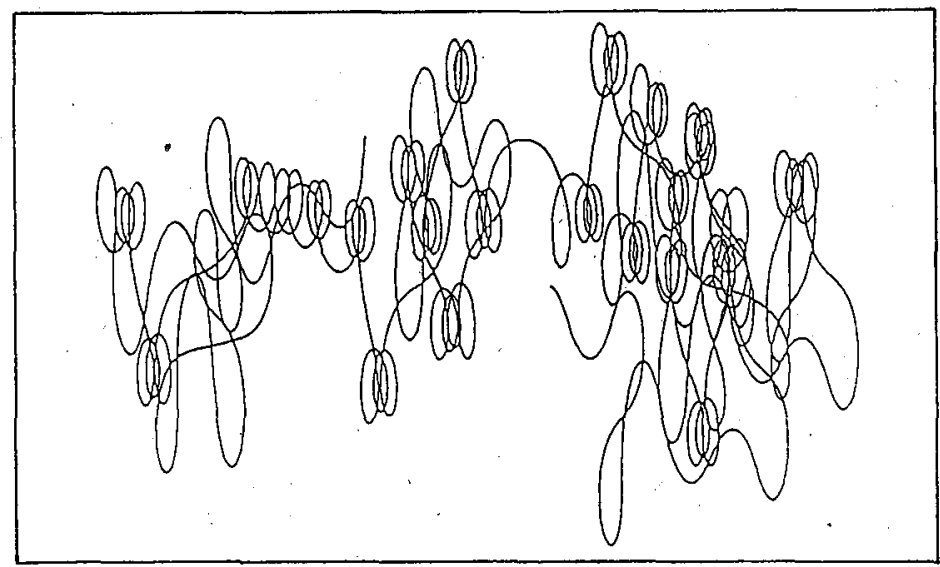

Fig. 3. Spacial entanglement of the dissipative elastica corresponding to a strange attractor in the dynamical analogy.

which was the motive behind trying to model some of these fluid motions using the elastica. In the case of a Hamiltonian system, the model was relatively straight forward and we found some interesting spacially chaotic deformation as shown in Fïg. 2. Some problems arise however in the spacial interpretation of positively and negatively dissipative elastica. In the case of positive disspation, i.e. damping, this may be interpreted as nonconservative tangential friction forces akin to the so called follower forces discussed in [5, 14]. Negative dissipative is consequently the adjoint system, a so called flutter set [14]. We may mention that the inclusion of this type of negative damping was motivated by some problems connected to protein deformation. Figures 2-4 show the results of our numerical experiments. The spacial entanglement which looks quite similar to randomly coiled polymer chains is quite interesting (Fig. 3). They correspond in the dynamical analogy to the region of a strange attractor in a parametrically excited system. However we feel that the most interesting numerical results are those with the spiral-like deformation (Fig. 4). They strongly resemble some of the pseudo-random Cornu-like spirals (Fig. 5) found for instance in the Riemann sums approximating oscillatory integral [11]. However they have some similarity to a well-known real physical phenomenon, namely the Bernard-Kàrmàn vortices (Fig. 6), [7, 8, 12, 13]. 


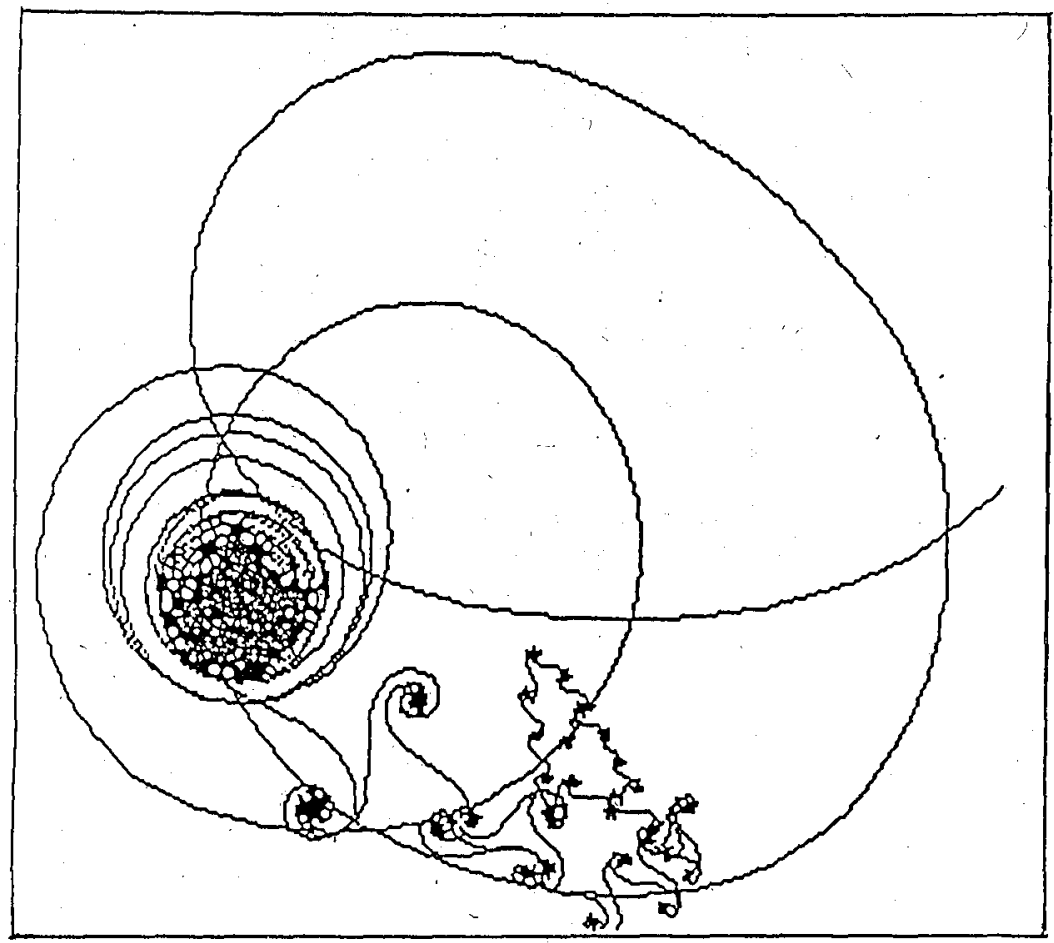

Fig. 4. Loop and spiral chaos in the imperfect flutter elastica.

(a)

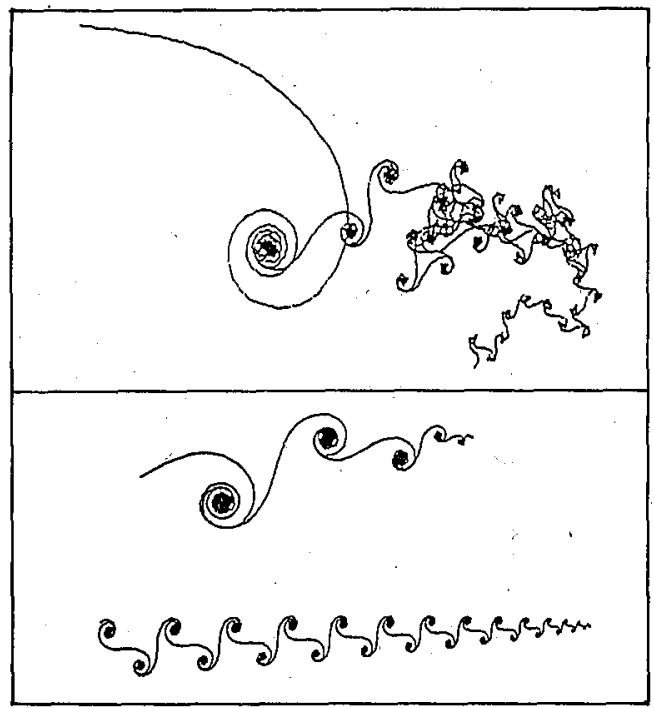

Fig. 5. Regülar (a) and irregular (b) Cornu-like spirals [11]. 


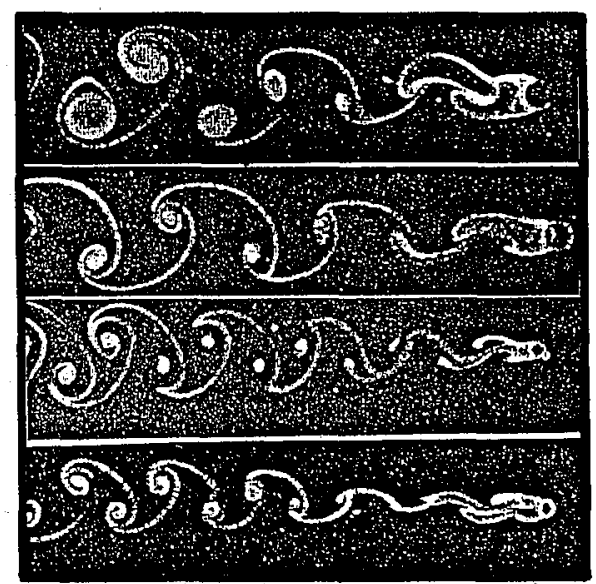

Fig. 6. Some experimental studies of vortex wake.

\section{Conclusion}

The elastica and in particular the imperfect flutter elastica provides a surprisingly simple model which reflects some fundamental aspects of diffusion and turbulence-like behaviour in fluids. Of course using our numerical technique we can never show true chaos. Nevertheless using the pre-entropy related ideas of $O$. Roessler we can show asymptotic chaos [2]. When we observed the spiral chaos of the elastica we were initially inclined to think that it is only a numerical instability phenomenon. However repeated independent calculations using different methods have convinced us that they are a true feature of the nonlinear dynamics of our model. The appearance of self-similarity on all scales which these spirals reflect shows that we are dealing with an important phenomenon which may be strongly linked to mixíng, diffusion-like process and chemical vortices.

\section{Appendix 1. The equation of the elastica}

1. The Hamiltonian of the perfect initially straight elastica corresponding to unforced undamped pendulum is

$$
\Phi^{\prime \prime}+\lambda^{2} \sin \Phi=0,
$$

where $\Phi$ is the angle of inclination of the central line of the elastica, $\left({ }^{\prime}\right)=\mathrm{d}() / \mathrm{d} s$ and $s$ is the arch length of the deformed elastica which is assumed to be totally inextensible. Consequently $\Phi^{\prime}$ is the curvature of the deformed (buckled) elastica. The parameter $\lambda=\sqrt{P / \alpha}$ corresponds to the natural frequency where $P$ is the axial load and $\alpha$ is the bending stiffness. This means that the bending moment is $M=\alpha \Phi^{\prime}$.

2. The imperfect elastica corresponding to a periodically excited pendulum is

$$
\Phi^{\prime \prime}+\lambda^{2} \sin \Phi=a \sin \omega s,
$$


where $a$ is a measure for the amplitude of the harmonic axial imperfection (crookedness) of the central line and $\omega$ is the frequency of this periodical spacial imperfection.

3. The elastica corresponding to a parametrically excited pendulum is

$$
\Phi^{\prime \prime}+\lambda^{2} \sin \Phi=a \sin \omega s \sin \Phi .
$$

4. The elastica corresponding to a parametrically excited pendulum with positive (or negative) damping is

$$
\Phi^{\prime \prime}+\lambda^{2} \sin \Phi \pm k \Phi^{\prime}=a \sin \omega s \sin \Phi,
$$

where $\mathrm{k}$ is the constant of linear positive (or negative) dissipation.

For more details and derivation see [4] or [14].

\section{Appendix 2. The differential equation of a fluid particle}

In addition to $x$ and $y$ we use a polar coordinate system $r, \varphi$ whose origin also coincides with the center of the cylinder, to describe the path $\varepsilon$ of the particle. The curvature of the path is thus given by

$$
\kappa=\frac{1}{R}=\frac{\mathrm{d} \Phi}{\mathrm{d} s}=\frac{\mathrm{d} \Phi}{\mathrm{d} y} \frac{\mathrm{d} y}{\mathrm{~d} s}=\frac{\mathrm{d} \Phi}{\mathrm{d} y} \sin \Phi,
$$

where $\Phi=2 \varphi$ is the slope of the path measured from the horizontal $x$ direction of the motion. On the other hand the streamline is given by

$$
\eta=y\left(1-\frac{a^{2}}{r^{2}}\right)
$$

and noting that $\sin \varphi=\sin \frac{\Phi}{2}=y / r$ we can eliminate $r$ and find

$$
\eta=y\left[1-\frac{a^{2}\left(\sin \frac{\Phi}{2}\right)^{2}}{y^{2}}\right] \text {. }
$$

This may be written as

$$
\left(\sin \frac{\Phi}{2}\right)^{2}=\frac{1}{a^{2}}\left(y^{2}-\eta y\right)
$$

and differentiating both sides as a function of $y$ one finds

$$
\left(\frac{\mathrm{d} \Phi / 2}{\mathrm{~d} y}\right)=\frac{1}{a^{2}}(2 y-\eta)
$$

That means

$$
\frac{1}{a^{2}}(2 y-\eta)=\sin \Phi \frac{\mathrm{d} \frac{\Phi}{2}}{\mathrm{~d} y}=\frac{1}{2} \sin \Phi \frac{\mathrm{d} \Phi}{\mathrm{d} y}
$$


or

$$
\frac{\mathrm{d} \Phi}{\mathrm{d} y} \sin \Phi=\frac{2}{a^{2}}(2 y-\eta) .
$$

From (1) and (7) it is evident that

$$
\frac{1}{R}=\Phi^{\prime}=\frac{2}{a^{2}}(2 y-\eta)
$$

where the constant $\eta$ gives the initial and final distance of the particle. Differentiating (8) one finds

$$
\Phi^{\prime \prime}=\left(\frac{4}{a^{2}}\right)\left(\frac{\mathrm{d} y}{\mathrm{~d} s}\right)
$$

Noting that $\mathrm{d} y / \mathrm{d} s=\sin \Phi$ we obtain

$$
\Phi^{\prime \prime}-\lambda^{2} \sin \Phi=0,
$$

where $\lambda=2 / a$. Equation (10) is mathematically identical with the equation of the elastica (Appendix 1).

\section{References}

[1] J.P. Eckmann, Rev. Mod. Phys. 53, 643 (1981).

[2] M.S. El Naschie, S. Al Athel, Naturforsch. A 44645 (1989).

[3] A. Mielke, P. Holmes, Arch. Ration. Mech. Anal. 101, 491 (1988).

[4] M.S. El Naschie, J. Phys. Soc. Jpn. 58, 4310 (1989).

[5] M.S. El Naschie, T. Kapitaniak, Phys. Lett. A (1990) in press.

[6] M.S. El Naschie, S. Al Athel, A.C. Walker, in: Nonlinear Dynamics in Engineering Systems, ed. W. Schiehlen, Springer Verlag, Berlin 1990, p. 67.

[7] H. Bernhard, Rev. Gen. Sci. Pur. Appl. 11, 1261, 1309 (1990).

[8] L. Prandtl, O. Tietjens, Fundamentals of Hydro- and Aerodynamics, Dover, New York 1957, (in particular pp. 64 and 211-213).

[9] K. Konno, M. Mituhasi, Y.H. Ichikawa, Chaos, Soliton and Fractals 1, 55 (1991).

[10] F. Homann, Forsch. Geb. Ingwes. 7, 423 (1939).

[11] E. Coutsias, N. Kazarinoff, Physica D 26, 295 (1987).

[12] H. Lamb, Hydrodynamics, Dover, New York 1945.

[13] L. Milne-Thomson, Theoretical Hydrodynamics, Macmillan, London 1960, (in particular pp. 233-237).

[14] M.S. El Naschie, Stress, Stability and Chaos, McGraw Hill, London 1990.

[15] J. Crank, The Mathematics of Diffusion, Clarendon Press, Oxford 1975.

[16] M.S. El Naschie, Int. J. Mech. Sci. 17, 387 (1975).

[17] M.S. El Naschie, in: 12th ASME Conference on Mechanical Vibration and Noise, Vol. No H0508E, ed. T.S. Sankar, American Society of Mechanical Engineers, New York 1989, p. 377. 